\title{
Evaluate the Effectiveness of Stress Management Training on Nurses' Stress
}

Akram Mahmoudian 1

Mahboubeh Heydari²

Leila Zolghadrnia ${ }^{3}$

1,2,3Alzahra university, Tehran, Iran

Doi:10.5901/mjss.2016.v7n3s2p228

\begin{abstract}
The aim of this study is to Evaluate the effectiveness of stress management training on nurses' stress. Cognitive-behavioral stress management program that was used in this study were able to reduce the scores of the experimental group. These findings are controversial with many of the results of previous studies. According to these reasons, it seems that the intervention program has failed to be effective in reducing nurses' stress, while the nurses' stress can be affected by other confounding variables during the research.
\end{abstract}

Keyword: stress, stress management training, nurses' stress

\section{Introduction}

One of the main problems in recent decades is the phenomenon of stress. This phenomenon has gained significance with the industrialized world and are widely and increasingly influenced employees (Tangri, 2003). Occupational stress is an expanding global experience and as a major risk factor in mental and physical illness, reduces work performance and job satisfaction as well (Levine et al., 2009). Because nursing is one of the occupations that involve spending long hours with people receiving services and they have a close relationship with other people, and are faced with incurable illness and diseases, hence they experience continuous severe psychological stress.

It has been recognized that the development of technical methods, processes and equipment alone are not effective in reducing injuries and accidents, and human factors such as mental characteristics have a significant role in this field (Concha-Barrientos et al., 2005). Occupational stress can underlie the events and occupational accidents and thus cause injuries to people and damage to equipment in the workplace. Studies on unsafe behaviors have shown that occupational stressors through poor concentration, distractibility, impaired memory, uncertainty and reducing in power of decision making contributed to the incidence of unsafe acts by employees (Goldenhare, 2003).

Nowadays, occupational stress play an important role in creating problems, physical disabilities and mental illnesses and staggering costs arising out of it and in respect of that, many studies have been done, especially about workers, nurses, midwives, teachers and administrators.

Because nurses have a sensitive job and deal with public health and their work in addition to their own health, it also has a direct impact on the health of other members of society; therefore, their occupational stress would be very expensive for organizations and may have undesirable effects such as desertion and absenteeism, which could directly or indirectly affect the quality of their work. The importance of this issue prompted researchers to study the effectiveness of stress management training on occupational stress of nurses.

\section{Methodology}

Design of present study is quasi-experimental design with pre-test and post-test and in general, it is an applied research. In this study, subjects were exposed to stress, then those who had a high score on the pretest, were divided into control and experimental groups. The experimental group were exposed to the independent variable and participated in the workshop of stress management training through the method of cognitive-behavioral therapy. After completing the course, both groups were tested and the effectiveness of stress management training on their occupational stress were determined. 
The study population consisted of all nurses working in Khatamolanbia Hospital in Tehran in 2014. For sampling, hospital wards were considered as classes and 3\% of each ward were selected by stratified sampling with an equal number inside the classes. After pre-test and calculating scores of stress, 30 people who earn high marks were chosen and randomly divided into two experimental and control groups. 3 nurses in the experimental group withdrew from further cooperation. Stress management training was carried out on 12 patients in the experimental group and after holding the class, so the post-test was conducted. In this study, due to the nature of the issue and use of a questionnaire including demographic information and Nursing Stress Scale proposed by Gray-Toft and Anderson used.

Demographic information includes information such as age, sex, marital status, education level, work shifts, overtime hours, employment status, job status and economic conditions.

Anderson Taft- Nursing Stress Scale (NSS) has been designed to measure the frequency and sources of stress experienced by nurses in the hospital. It contains 34 questions and analyzes the stress factors in seven areas which as follows: 7 questions about the suffering and death of patients, 5 questions about conflict with physicians, 3 questions about lack of knowledge in dealing with emotional needs of patients and their families, 3 questions about the lack of social support, 7 questions about conflicts with other nurses and supervisors, 6 questions about workload, 5 questions about uncertainty of treatment. Based on a Likert-scale, type of response is set in four ranges: from never to very much and based on their understanding of what happened in the workplace. Scores below 39 indicate low stress, scores between 40 to 62 show moderate stress and scores higher than 63 indicates high stress.

Earlier in the message, validity and reliability of these instruments have been approved. Thus, the reliability of the instrument was approved by 11 professors of the School of Nursing and Midwifery, Iranian University of Medical Sciences. To ensure the validity of the test-retest reliability was obtained r=87 (Sherbaf Nejad, 2008) and Cronbach's alpha coefficient of this test is 0.93 (Hussaini, 2011). In this study, to determine the validity, the questionnaire was given to 18 nurses who were selected randomly and its validity was confirmed. To evaluate the reliability of the questionnaire through internal consistency with Cronbach's alpha coefficient was conducted inquiries. Accordingly internal consistency of Toft- Anderson's nurses' stress scale (0.91) were calculated and confirmed.

\section{Findings}

Demographic characteristics of two experimental and control groups are presented in Table 1.

Table 1. The volume of the sample group according to gender and ward

\begin{tabular}{|c|c|c|c|c|c|c|}
\hline \multirow{2}{*}{ No. } & \multirow{2}{*}{ Variable } & \multirow{2}{*}{ Sub-Variable } & \multicolumn{2}{|c|}{ Experimental group } & \multicolumn{2}{c|}{ Control group } \\
\cline { 4 - 7 } & & Frequency & Percent & Frequency & Percent \\
\hline \multirow{3}{*}{1} & \multirow{3}{*}{ Sex } & Female & 5 & $41 / 7$ & 9 & 60 \\
\cline { 3 - 7 } & & Man & 7 & $58 / 3$ & 6 & 40 \\
\cline { 3 - 7 } & \multirow{2}{*}{1} & Total & 12 & 100 & 15 & 100 \\
\cline { 3 - 7 } & & Special & 5 & $41 / 7$ & 5 & 33.33 \\
\cline { 3 - 7 } & & Ordinary & 7 & $58 / 3$ & 10 & 66.66 \\
\hline & \multicolumn{2}{|c|}{ Total } & 12 & 100 & 15 & 100 \\
\hline
\end{tabular}

Mean and standard deviation in scores of pre-test and post-test of experimental and control groups are presented in Table 2.

Table 2. The mean and standard deviation of the studied variables

\begin{tabular}{|c|c|c|c|c|c|c|c|c|}
\hline \multirow{2}{*}{ Variables } & $\begin{array}{c}\text { Pre-test in the experimental } \\
\text { group }\end{array}$ & \multicolumn{2}{|c|}{$\begin{array}{c}\text { Posttest in the } \\
\text { experimental group }\end{array}$} & \multicolumn{2}{c|}{$\begin{array}{c}\text { Pre-test in the control } \\
\text { group }\end{array}$} & \multicolumn{2}{|c|}{$\begin{array}{c}\text { Posttest in the control } \\
\text { group }\end{array}$} \\
\cline { 2 - 8 } & Mean & Standard deviation & Mean & Standard deviation & Mean & Standard deviation & Mean & Standard deviation \\
\hline Stress & 59.67 & 15.80 & 55 & 14.31 & 48 & 11.66 & 47.60 & 10.7 \\
\hline
\end{tabular}

To test the hypotheses, analysis of variance and bivariate ANOVA were used.

Pre-test score was considered as a covariate and its effect on the post test scores was controlled by using analysis of covariance. Since the analysis of covariance is a parametric test, the assumptions of normal distribution and equality of variances were studied. The results of the statistical assumptions showed that both preconditions for the equality of 
variance and normal distribution are established. The analysis of the effect of stress management training on stress scores of experimental and control groups in post-test can be seen in table 3 :

Table 3. Analysis of the effect of stress management training on nurses' stress

\begin{tabular}{|c|c|c|c|c|c|}
\hline Sources of variance & Sum of the squares & Degrees of freedom & average of squares & $\mathrm{F}$ & $\mathrm{P}$ \\
\hline Stress & 348.30 & 1 & 348.30 & 26.5 & 0.0001 \\
\hline Group & 44.44 & 1 & 43.44 & 3.38 & 0.78 \\
\hline Error & 320.25 & 23 & 13.14 & & \\
\hline Total & 674.39 & 26 & & & \\
\hline
\end{tabular}

As can be seen in Table 3, the amount $F$ and the impact of covariate (26.5) is significant, because its probability is close to zero and is smaller than the significance level of 0.05 . The seventh assumption that was the correlation between independent and covariate, been observed. But the influence of the independent variable $F$ (Group), (3.38) is not significant. After removing the effect of pretest, there is no significant difference between average scores in experimental and control groups in the stress post-test. The impact of stress management training on nurses' stress is different according to sex and ward and table 4 compared the means of these two variables.

Table 4. Comparing the means of stress in both sexes and ward

\begin{tabular}{|l|l|l|c|c|c|}
\hline Sex & Ward & Group & Mean & Standard deviation & Number \\
\hline \multirow{4}{*}{ Man } & \multirow{3}{*}{ Ordinary } & Experiment & 35 & - & 1 \\
\cline { 2 - 6 } & & Control & 41.5 & 6.41 & 6 \\
\cline { 2 - 6 } & \multirow{2}{*}{ Special } & Experiment & 52.25 & 9.43 & 4 \\
\cline { 3 - 6 } & \multirow{3}{*}{ Woman } & Control & 58.33 & 6.5 & 6 \\
\hline \multirow{3}{*}{ Ordinary } & Experiment & 58 & 16.33 & 4 \\
\cline { 2 - 6 } & & Control & 52.75 & 12.84 & 2 \\
\cline { 2 - 6 } & \multirow{2}{*}{ Special } & Experiment & 68 & - & 2 \\
\cline { 3 - 6 } & & Control & 39.5 & 3.53 & \\
\hline
\end{tabular}

Bivariate ANOVA results for the stress control and experimental groups in both genders and two wards are shown in Table 4.

Table 5. Bivariate ANOVA for sex and ward

\begin{tabular}{|c|c|c|c|c|c|}
\hline Sources of variance & Sum of the squares & Degrees of freedom & average of squares & $\mathrm{F}$ & $\mathrm{P}$ \\
\hline Stress & 3.19 & 1 & 3.19 & 0.3 & 0.59 \\
\hline Group & 25.19 & 1 & 25.19 & 2.36 & 0.14 \\
\hline Error & 20.08 & 1 & 20.08 & 1.88 & 0.18 \\
\hline Total & 191.62 & 23 & 8.33 & & \\
\hline
\end{tabular}

According to Table 5, the main effect of sex on stress is not significant. The amount of $F(0.3)$ with degrees of freedom 1 and 23 is smaller than the amount of the table (4.27) at the 0.05 level. So one can say with $95 \%$ confidence that there is no significant difference between the two sexes in stress and the null hypothesis is not rejected.

The main effect of ward on stress is not significant. The amount of $F(2.36)$ with degrees of freedom 1 and 23 is smaller than the amount of the table (4.27) at the 0.05 level. So one can say with $95 \%$ confidence that there is no significant difference between the two wards in stress and the null hypothesis is not rejected.

The interaction effect between sex and ward on stress is not significant. The amount of $F$ with degrees of freedom 1 and 23 is smaller than the amount of the table (4.27) at the 0.05 level. So one can say with $95 \%$ confidence that there is no significant difference between the interaction effect between sex and ward in stress and the null hypothesis is not rejected. 


\section{Discussion and Conclusion}

Cognitive-behavioral stress management program that was used in this study were able to reduce the scores of the experimental group. These findings are controversial with many of the results of previous studies.

To explain these findings, we can refer to the limitations that existed in this study. Some nurses working in many different shifts or in other centers which may increase the occupational stress and the researcher cannot control these things. Due to the large number of questionnaires and questions, nurses maybe not accurate enough to answer the questions related to occupational stress which were listed in the final questionnaire. This carelessness can result from business and exhaustion. The results of stress management programs have little effect in reducing the stress level of research samples in some previous studies (Shakernia, 2012), which can be influenced by the control variables and other disturbing variables that affect the research.

According to researchers, managers have an important role to eliminate or reduce stressors in nurses. These factors are effective in reducing job stress: the elimination of paperwork, assigning these tasks to the secretary, briefing courses, in-service training courses and addressing the shortage of nurses. According to these reasons, it seems that the intervention program has failed to be effective in reducing nurses' stress, while the nurses' stress can be affected by other confounding variables during the research.

The effectiveness of stress management program on stress reduction for male and female nurses were the same and the performance is satisfactory. The effectiveness of this program on stress reduction in different wards of hospital were the same and the performance is satisfactory for both ordinary and special wards. Letvak and Buck (2008) showed that female nurses compared with male nurses have higher scores in terms of occupational stress. Recent research proves that nurses, especially in clinical units are at high risk of stress and stress is more prominent in intensive care units. Whereas the results of research conducted by Kane et al and Hipwell et al. shows that nurses who work in intensive care units, do not necessarily have a high degree of stress or burnout (Matrunola, 1999, p. 828, quoted by E'temadi, 2004). One feature of the program was to reduce the occupational stress related to each part separately and using cognitive and behavioral techniques during regular meetings. On the other hand, the participation of nurses working in different wards and recounting stresses in groups, can play a role of a catalyst and reduce some of the negative thoughts and cognitive errors. It seems that the program is now able to increase interaction between staff in different wards in the form of group meetings and indirectly encourage them to share their concerns with the work. Dealing with stress was the same in both sexes and the stated reason was not apparently effective intervention.

This study had several limitations. Although Toft-Anderson's nurses' stress Scale (NSS) is standardized and its validity has been confirmed, but like all human sciences, it is dependent on culture and does not apply in all societies, cultures, organizations and conditions. One of the weaknesses of self-report questionnaires, different perceptions that people have from the words and sentences and sometimes it can be caused inconsistent results; Therefore, differences in nurses' perceptions of the implications of the questionnaire in response to questions is another limitation of the study.

\section{References}

E'temadi, S. (2005). Comparing the relationship between occupational stress and job satisfaction in CCU unit nurses and ordinary unit nurses in the university teaching hospital of Tehran and Iran, master's degree thesis, psychology, Al-Zahra University.

Shakernia, A. (2012). The relationship between moral confusion, psychological hardiness and burnout among nurses in Rasht city. Journal of Ethics and History of Medicine. Volume 4

Antoni M H, Ironson G, Schnneiderman, N. (2007). Cognitive Behavioral Stress Management. Alemohammad, SJ, Jokar S, Neshat Doost H.(Translators).315. In Press. (Full Text in Persian).

Concha-Barrientos, M., Nelson, D. I., Fingerhut, M., Driscoll, T., \& Ligh, J. (2005).The global burden due to occupational injury.American Journal of Industrial Medicine, 48(6), 470-81.

Goldenhar, K. (2003). Modeling relationships between job stressors and injury and near- miss outcomes for construction laborers. Work \& Stress, 17(3), 218- 40.

Gray-Toft P, Anderson J. (1981). The nursing stress scale: Development of an instrument. J Psychopathol Behav Assess, 3(1): 11-23

Levine, J. A., Pavlidis, I. T., MacBride, L., Zhu, Z., \& Tsiamyrtzis, P. (2009). Description and clinical studies of a device for the instantaneous detection of office-place stress. Work: A Journal of Prevention, Assessment and Rehabilitation, 34(3), 359-64.

Tangri, R.P. (2003). What stress costs. Halifax: Chrysalis performance strategies inc. 